\title{
Strain-Specific Symbiotic Genes: A New Level of Control in the Intracellular Accommodation of Rhizobia Within Legume Nodule Cells
}

\author{
Benjamin Gourion, ${ }^{1,+}$ and Benoit Alunni2, ${ }^{2,}$ \\ 'LIPM, Université de Toulouse, INRA, CNRS, Castanet-Tolosan, France; and ${ }^{2}$ Institute for Integrative Biology of the Cell, UMR \\ 9198, CNRS/Université Paris-Sud/CEA, Gif-sur-Yvette, France
}

Accepted 14 January 2018.

Interactions between rhizobia and their legume hosts involve the coordination of bacterial infection and nodule organogenesis. These interactions are generally considered as highly specific with a given legume species being only nodulated by a restricted number of rhizobial species or strains. A major breakthrough has been made in the understanding of the molecular basis of this specificity with the discovery, in the last 40 years, of Nod factors and their biosynthetic genes in rhizobia and of cognate receptors and downstream signaling in plants. However, many rhizobiumlegume interactions result in nodules that ultimately display ineffective or no nitrogen fixation (Fix ${ }^{-}$phenotype), suggesting that other regulatory mechanisms are involved in the establishment of a functional nodule, acting after bacteria have been internalized into plant cells. Since the 1950s, plant lines have been obtained that show strain-specific nonfixing nodules in different legume species, such as soybean, pea, vetch, and clover (Tirichine et al. 2000), although the first genes responsible for this phenotype have been cloned in model legumes only recently (Wang et al. 2018).

In a very recent set of papers, three of such plant genes were identified and characterized: APN1 in Lotus japonicus and NFS1 and NFS2 in Medicago truncatula. The apn1 mutant of L. japonicus (Yamaya-Ito et al. 2018) displays nodules that are similar to the wild type and are able to fix nitrogen upon inoculation with some Mesorhizobium loti strains, including the model MAFF303099. In contrast, the apn1 mutant is Fix ${ }^{-}$upon inoculation with the strain TONO of Mesorhizobium loti. In such a situation, the nodules are smaller and often dark brown, suggesting an activation of plant defense reactions. Remarkably, even in those nodules, the plant cells are correctly infected but bacteroids (the intracellular form of rhizobia) degenerate prematurely. The responsible gene encodes an aspartic peptidase harboring a signal peptide and is expressed specifically within infected cells of the nodules, suggesting a possible targeting to the symbiosome (the vesicles containing bacteroids). At this step, the precise role and

${ }^{\dagger}$ Corresponding authors: Benjamin Gourion;

E-mail: benjamin.gourion@toulouse.inra.fr and

Benoit Alunni; E-mail: benoit.alunni@i2bc.paris-saclay

Funding: This work was funded by a Labex TULIP grant (ANR-10-LABX-41, ANR-11-IDEX-0002-02), Labex Saclay Plant Science grant (ANR-10-LABX0040-SPS), and Agence Nationale de la Recherche grant (ANR-17-CE200011-02, ANR-17-CE20-0013).

This article is in the public domain and not copyrightable. It may be freely reprinted with customary crediting of the source. The American Phytopathological Society, 2018. molecular targets (i.e., plant or bacterial proteins or both) of this peptidase in controlling rhizobial accommodation remain unclear.

The NFS1 and NFS2 genes have been formerly identified as a single locus (Mtsym6) in a small mapping population of Medicago truncatula conferring strain-specific Fix ${ }^{-}$nodules (Tirichine et al. 2000). Careful reexamination of this locus in a sister background (Jemalong A17 being similar to Jemalong 6, used by Tirichine et al. [2000]) allowed the identification of two genes, NFS1 and $N F S 2$, that restrict the nodulation of M. truncatula A17 by two strains of Sinorhizobium meliloti (i.e., A145 and Rm41), whereas the same strains induce Fix+ nodules on M. truncatula DZA315 (Wang et al. 2017, 2018; Yang et al. 2017). In each mutant, bacteroids die in planta and the nodules display early senescence. Cloning of the two genes revealed that they encode members of the nodule-specific cysteine-rich (NCR) peptide family (approximately 600 genes in the $M$. truncatula genome). The precise role of these symbiosome-targeted defensin-like antimicrobial peptides is not yet fully understood. Nevertheless, they are responsible for dramatic morphological and physiological changes in the microsymbiont, called terminal bacteroid differentiation (TBD), a step that is required for subsequent nitrogen fixation in some legume clades (Alunni and Gourion 2016). In vitro, NCR peptides can induce membrane permeability, and bacterial sensitivity to these peptides strongly depends on the structure of the envelope and surrounding polysaccharides. Moreover, antimicrobial peptides may be toxic for bacteria, and, in the case of nonadapted strains, they may be cleared from the nodule by NCRs, as these strains cannot survive TBD when NFS1 and NFS2 are expressed. Finally, this strain-specific effect of NCR genes prompted the authors to suggest that NCR genes may be actors of the coevolution process ongoing between rhizobial strains and plant genotypes and that rapid spreading of the NCR family in the Medicago genome could be part of this scenario (Wang et al. 2018).

Given that former strain-specific Fix ${ }^{-}$plant genotypes have been identified in plants closely related to genus Medicago (pea, vetch, and clover are NCR-producing plants), one could suspect these lines to be affected in a NCR gene. However, the identification of apnl in Lotus japonicus and of soybean lines displaying a similar phenotype opens new questions regarding the processes that occur in plants that do not produce NCRs and that do not induce TBD. Also, an APN1 homolog was identified in Medicago spp., opening the possibility that two distinct and independent mechanisms control the late recognition process in the IRLC clade. These molecular mechanisms remain to be described. The study of the newly identified plant determinants of strain specificity might be facilitated by the previous identification 
of some of their counterparts in rhizobia (Schumpp and Deakin 2010). Such factors include bacterial exopolysaccharides, in which structural changes could affect NCR action (Simsek et al. 2007), and a protease that has been shown to be able to process NCR peptides in vitro (Price et al. 2015). Now, the challenge will be to establish the correspondence between rhizobial and legumes factors involved in these late recognition processes. To achieve this, the previous characterization of compatibility levels for many strains and lines in the Sinorhizobium-Medicago model system (Crook et al. 2012) will be a powerful tool.

Whatever the molecular basis of the strain discrimination, the identification of these new actors stresses the negative role that plant immunity can have on rhizobia-legume interactions, as NCRs display similarity to defensins and alteration of APN1 induces the development of plant defense reactions. It is also noteworthy that many Fix ${ }^{-}$mutants may actually be strain-specific, though they have not yet been tested for this phenotype, as most laboratories use only one model strain to phenotype plant mutants. Finally, these reports have highlighted the importance of understanding the late steps of the symbiotic process. This will be particularly crucial for those who want to transfer nodulation to nonlegume crops. So, in addition to the transfer of early recognition/signaling modules leading to organogenesis and infection, this new layer of interaction specificity will have to be considered to achieve functional nodulation in cereals and other nonlegume crops.

\section{LITERATURE CITED}

Alunni, B., and Gourion, B. 2016. Terminal bacteroid differentiation in the legume-rhizobium symbiosis: Nodule-specific cysteine-rich peptides and beyond. New Phytol. 211:411-417.
Crook, M. B., Lindsay, D. P., Biggs, M. B., Bentley, J. S., Price, J. C., Clement, S. C., Clement, M. J., Long, S. R., and Griffitts, J. S. 2012. Rhizobial plasmids that cause impaired symbiotic nitrogen fixation and enhanced host invasion. Mol. Plant-Microbe Interact 25:1026-1033.

Price, P. A., Tanner, H. R., Dillon, B. A., Shabab, M., Walker, G. C., and Griffitts, J. S. 2015. Rhizobial peptidase HrrP cleaves host-encoded signaling peptides and mediates symbiotic compatibility. Proc. Natl. Acad. Sci. U.S.A. 112:15244-15249.

Schumpp, O., and Deakin, W. J. 2010. How inefficient rhizobia prolong their existence within nodules. Trends Plant Sci. 15:189-195.

Simsek, S., Ojanen-Reuhs, T., Stephens, S. B., and Reuhs, B. L. 2007. Strain-ecotype specificity in Sinorhizobium meliloti-Medicago truncatula symbiosis is correlated to succinoglycan oligosaccharide structure. J. Bacteriol. 189:7733-7740.

Tirichine, L., de Billy, F., and Huguet, T. 2000. Mtsym6, a gene conditioning Sinorhizobium strain-specific nitrogen fixation in Medicago truncatula. Plant Physiol. 123:845-851.

Wang, Q., Liu, J., Li, H., Yang, S., Körmöczi, P., Kereszt, A., and Zhu, H. 2018. Nodule-specific cysteine-rich peptides negatively regulate nitrogenfixing symbiosis in a strain-specific manner in Medicago truncatula. Mol. Plant Microbe Interact. 31:240-248.

Wang, Q., Yang, S., Liu, J., Terecskei, K., Ábrahám, E., Gombár, A., Domonkos, A., Szúcs, A., Körmöczi, P., Wang, T., Fodor, L., Mao, L., Fei, Z., Kondorosi, É., Kaló, P., Kereszt, A., and Zhu, H. 2017. Hostsecreted antimicrobial peptide enforces symbiotic selectivity in Medicago truncatula. Proc. Natl. Acad. Sci. U.S.A. 114:6854-6859.

Yamaya-Ito, H., Shimoda, Y., Hakoyama, T., Sato, S., Kaneko, T., Hossain, M. S., Shibata, S., Kawaguchi, M., Hayashi, M., Kouchi, H., and Umehara, Y 2018. Loss-of-function of ASPARTIC PEPTIDASE NODULE-INDUCED 1 (APN1) in Lotus japonicus restricts efficient nitrogen-fixing symbiosis with specific Mesorhizobium loti strains. Plant J. 93:5-16.

Yang, S., Wang, Q., Fedorova, E., Liu, J., Qin, Q., Zheng, Q., Price, P. A., Pan, H., Wang, D., Griffitts, J. S., Bisseling, T., and Zhu, H. 2017. Microsymbiont discrimination mediated by a host-secreted peptide in Medicago truncatula. Proc. Natl. Acad. Sci. U.S.A. 114:6848-6853. 